Military Technical College

Kobry El-Kobba

Cairo, Egypt

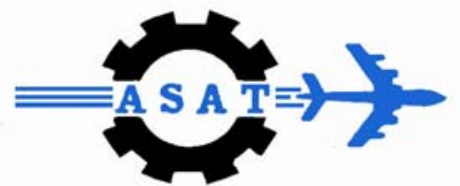

12-th International Conference

on

Aerospace Sciences \&

Aviation Technology

\title{
NUMERICAL MODELING OF THE INTERFACIAL INSTABILITY IN TWO-PHASE FLOWS CONSIDERING BUOYANCY AND CAPILLARY PHENOMENA
}

\author{
BALABEL, ${ }^{1 *}$ A. and HEGAB ${ }^{2}$, A. M.
}

\begin{abstract}
The overall objective of the present paper is to develop an accurate and robust numerical modeling of the instability of an interface separating two fluids (liquid-gas) due to buoyancy and capillary effects. The governing unsteady Navier-Stokes along with the stress balance and kinematic conditions at the interface are solved separately in each fluid using the finite-volume approach. The present numerical model interprets the surface and the body forces as a boundary value conditions on the interface. Thus enables accurate modeling of fluid flows driven by either body or surface forces. The position of the interface is captured implicitly on the Eulerian grid by the zero level set function, while appropriate interpolations at the interface are used to enforce the associated jump conditions. To asses the developed numerical model and its versatility, a selection of different unsteady tests are examined: oscillation of a capillary wave, sloshing in a rectangular tank, and the broken-dam problem involving different density fluids. The computational results demonstrate a remarkable capability of the developed numerical model to predict the dynamical characteristics of the two-phase flows, which is of great importance in many industrial and engineering applications.
\end{abstract}

Keywords: interfacial instability, two-phase flow, level set method, capillary wave, sloshing, broken dam.

\section{INTRODUCTION}

The instability problems of an interface separating two fluids with different properties remain a considerable challenge to mathematicians and numerical analysts alike. Such problems are encountered in hydrodynamics and in the theory of convective heat and mass transfer in two-fluid systems, where the interaction processes contribute significantly to momentum and energy exchanges. Detailed analysis of these processes with numerical models may aid in understanding the

$1^{*}$ Lecturer, Dpt. of Mech. Power Engineering, Faculty of Engineering, Menoufia University, Shebin Elkom, Egypt.

2* Associate Professor, Dpt. of Mech. Power Engineering, Faculty of Engineering, Menoufia University, Shebin Elkom, Egypt. 
physical processes of two-phase flows. These range from rather small scale phenomena, such as the breakup or coalescence of raindrops, to large scale flows, like the motion of fuel in a space vehicle under low gravity conditions. Laboratory investigations of these phenomena are often complicated especially by problems encountered in simulating the actual physical situation. Therefore, a great attention has recently turned to this type of instability in connection of a variety of fluid flow problems particularly with the recent progress in computer performance. Accordingly, the superior variability of carefully executed simulations with possible resolution in such fields can virtually replace experiments and provides us with an important source of information that is not available experimentally [1].

The capillary phenomena, caused by the existence of surface tension on an interface separating two fluids, may occur when the interface possesses considerable curvature or due to a local variation of surface tension. In both cases, interfacial forces are developed that change the nature of the motion in each phase or induce motion originally absent. Formally, that appears in a change of the boundary conditions on the interface. Consequently, the effect of surface tension on the hydrodynamics and also on the basic mechanism for thermocapillary instability in a two-fluid system is considered to play a fundamental role in many natural and industrial phenomena. (e. g. interaction processes between air and ocean with breaking waves, crystal growth processes that are commonly used in microelectronic industry). There are several outstanding papers [2, 3 and 4] describing the early research, the detail derivation of basic equations, and the complexity of the physical phenomena included.

In general, the effect of either the buoyancy or the capillary forces in two fluidsystems plays an important role in a large domain of phenomena in physics and physical chemistry where the hydrodynamical regimes in such systems depend on the heat and mass transfer processes occurring in the systems. The key feature of these phenomena is the interaction between the interfacial forces at the surface of fluid layers and the fluid inside. If the fluid is incompressible, these forces immediately affect the motion of the entire fluid; in turn, this motion changes the configuration of the boundary which determines the forces. This can be considered as a type of force-fluid interaction problems.

Recently, there has been a strong interest in developing robust and accurate numerical methods for simulating complex fluid/fluid flows with imbedded interface of discontinuity. Such flows typically involve discontinuous material properties across the interface, which could pose severe limitation in the accuracy of common CFD methods. Moreover, and according to the basic principles of hydrodynamics, the condition of a balance of the forces acting in each phase either in the normal or the tangential directions of the interface must be fulfilled. Accordingly, the numerical methods for such problems engage two essential problems. On one hand, it is difficult to maintain the conservation properties of fluid/fluid system. On the other hand, the character of the interface can reduce the efficiency and the accuracy of iterative solution methods.

There is a class of fluid/fluid problems in which the interface between the fluids can be considered as a free-boundary, and therefore the computation can be limited to the more viscous and dense fluid. The idea of solving only one fluid and use of 
appropriate boundary conditions at the free surface has been used with almost any interface tracking method such level set method (LSM) or volume of fluid (VOF) method to capture the dynamic evolution of the moving interface. In such formulation, surface tension effects are modeled classically by positing a pressure jump at the interface that is proportional to the local curvature (the Laplace-Young condition). Therefore, the computation of the pressure can be done in a standard way without pressure and velocity oscillations at the interface that are common in two-fluid systems with large density ratios. However, to reduce the associated numerical diffusion which will destroy the sharpness of the interface, several algorithms have been presented (e.g. [7, 8 and 9]). An extended review of recent advances in computations of incompressible flows involving a fully nonlinear free surface that treated explicitly as a discontinuity is given in [1].

In the second class of fluid/fluid problems, both fluids are solved either as a continuous medium or they are treated separately. In the former approach, the governing equations are solved within each fluid with smoothed, instead of discontinuously changed, fluid properties across the interface by using the Heaviside function formulation as made in [10]. The interfacial boundary conditions or jump conditions can be integrated into the equation of motion, resulting in a body force concentrated in the interface of a single incompressible fluid with variable properties [11]. Thus the interface is smoothed across a finite thickness region, usually a few grid points thick introducing a transition region. This results in smearing of the flow properties and variables, forcing them to be continuous at the interface regardless of the appropriate jump conditions. Consequently, the most prominent methods of [11, 12] induce the so-called spurious or "parasitic" currents as a result of inconsistent modeling of the surface tension effects and the associated jump conditions. These numerical artifacts may lead in some cases to catastrophic instability in interface calculations and only moderate accuracy in regarding to determining the curvature and the steep gradients occurring at the interface. These problems are often compounded by the presence of multiphase flow problems involve fluids with very high density and viscosity ratios. Many numerical methods have been developed to overcome these problems (e.g. [13, 14]), however, this results in additional computational cost that can be very demanding in large 3-D computations.

In the present study, we apply the second approach for computing fluid/fluid problems, in which the motion of each phase is separately treated. This method has an important advantage of not limiting the density ratio between two phases, and hence easily allows for large density ratio of water to air, while maintaining high numerical stability [3]. The interfacial boundary conditions, composed of kinematic and dynamical conditions, are satisfied at the interface between the two phases. The surface tension force is formulated as a gradient of a potential present only at the interface. Therefore both the surface tension potential and the pressure in the momentum equations are dynamically similar, and the physical pressure drop across the interface must exactly cancel the surface tension forces. Preliminary aspects of this work were described in [15].

In order to describe the evolution of topologically moving interfaces, a number of methods have been proposed so far. In the context of incompressible flow, there are two numerical approaches for solving fluid/fluid problems. The first approach is based on the explicitly tracking the interface, e. g. the boundary integral method [16] 
and the Lagrangian methods [17], while in the second approach, known as Eulerian approach, the interface is implicitly captured by a scalar function with fixed grids covering the two fluids regions. Thus, the calculation is conducted stably no matter how complicated the interface evolves. Furthermore, the extension from 2-D to 3-D can be achieved easily, especially in the presence of merging and breaking of interfaces. In this category, the marker and cell (MAC) method [7], VOF method [8] and LSM [10] have been widely accepted. The level set methods currently attract considerable attention. Since LSM only requires solution of a scalar hyperbolic transport equation, it is simple from an algorithmic point of view, its implementation is straightforward and the computational effort is negligible in comparison to VOF method. Therefore, LSM is adopted in the present work.

In the present paper, we extend further the approach of separately treating fluids to include all the surface forces arising in the normal direction to the interface due to surface tension effects. The buoyancy effect is also modeled in a similar way to the surface tension effect. Consequently, the dynamical and kinematic conditions between the two phases are defined exactly at the interface avoiding the visual errors associated with nonphysically smearing out of the discontinuous material properties across the interface. An extended level set method for tracking the moving interfaces is presented, where the interface is modeled as the zero level set of a smooth function defined entirely in both sides of the interface. The governing equations for both phases are solved using the projection method on a nonstaggered grid system, constructed in the physical domain rather than using any domain mapping.

The outline of the paper is as follows: first the governing equations and their solution algorithm are described with a review of the level set method. Next, all the particulars of the numerical modeling of the surface and body forces are described. Finally, the effectiveness and usefulness of the present scheme are evaluated qualitatively and quantitatively in a wide rang of numerical example.

\section{COMPUTATIONAL METHODOLOGY}

\section{1- Governing Equations}

In two-phase problems, the Navier-Stokes equations have to be satisfied in each fluid. Then, the flow is governed by the following equations written in the primitive dependent variables as:

$$
\begin{gathered}
\nabla \cdot \mathrm{u}_{k}=0 \\
\frac{\partial \mathrm{u}_{\mathrm{k}}}{\partial t}+\left(\mathrm{u}_{k} \cdot \nabla\right) \mathrm{u}_{k}=-\frac{1}{\rho_{k}} \nabla p_{k}+v_{k} \nabla^{2} \mathrm{u}_{k}
\end{gathered}
$$

where the subscript $k$ means the quantities in fluid $k$ for $k=1,2$ corresponding to the liquid and gas phases, respectively. The quantities $\mathbf{u}, p, \rho$ and $v$ denote velocity vector, pressure, density and kinematic viscosity. In the above equations, it is noticed that the terms represent the gravitational field and the surface tension are 
included in the pressure term through the interfacial boundary conditions as described later.

\section{2- Interfacial Boundary Conditions}

The boundary conditions at the interface, or jump conditions, are comprised of the dynamic and kinematic conditions. In the case of two immiscible fluids, referring to figure (1), the dynamically conditions may be written in the following general form:

$$
[-p \mathrm{I}+2 \mu \mathrm{D}] . \mathrm{n}=-\left(\sigma \mathrm{nn}+\nabla_{t} \sigma\right)
$$

where $I$ is the identity matrix, $p$ is the pressure, $\mu$ is the dynamic viscosity, $\sigma$ is the surface tension coefficient, and $D$ is the deformation tensor whose components are $\mathrm{D}_{\mathrm{ij}}=0.5\left(\mathrm{u}_{\mathrm{i}, \mathrm{j}}+\mathrm{u}_{\mathrm{j}, \mathrm{i}}\right)$. The bracket means the jump of the stresses along the fluid interface

, the unit normal vector $\mathbf{n}$ is taken from fluid 2 to fluid 1 and $\mathbf{t}$ is an arbitrary vector perpendicular to the normal to the interface. The curvature of the interface is computed from

$$
\kappa=\nabla \cdot \mathrm{n}
$$

The normal and tangential velocity components are $V_{n}$ and $V_{t}$ respectively. It is clearly that, the effect of the surface tension is to balance the jump of the normal stress along the fluid interface. The second term on the right hand side of the above equation is the stress due to gradient on surface tension in the local interface coordinates or Marangoni effect, usually important when a temperature gradient is applied parallel to the interface, e. g. thermocapillary convection. The interfacial curvature $\kappa$ is computed from:

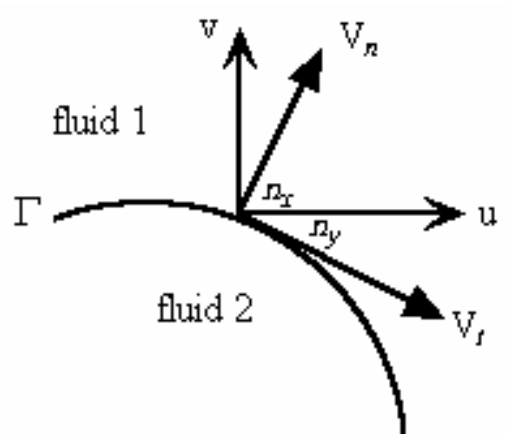

Figure (1) Schematic of normal and tangential velocity components of an arbitrary interface

In contrast to the previous two-phase numerical methods [10], in which the interfacial jump conditions are embedded naturally on the formulation, the jump conditions at the interface between the two fluids, described by Eq. (3), are treated here as a boundary conditions enforced explicitly at the interface. Taking the projections of the jump conditions in the directions normal and tangential to the interface, considering a constant surface tension, one obtains the following two equations in the normal and tangential directions, respectively: 


$$
\begin{gathered}
{[p-2 \mu(\nabla \mathrm{u} \cdot \mathrm{n}) \cdot \mathrm{n}]=\sigma \mathrm{m}} \\
{[\mu(\nabla \mathrm{u} \cdot \mathrm{n}) \cdot \mathrm{t}+\mu(\nabla \mathrm{u} \cdot \mathrm{t}) \cdot \mathrm{n}]=0}
\end{gathered}
$$

It is noticed from the above equations that surface tension effects are included in the normal stress balance, Eq. (5), while the equality of the shear stress is satisfied in the tangential direction, Eq. (6).

In case of considering the buoyancy effect, the gravity is assumed to be lumped with the pressure to define a piezometric pressure; $p=\rho g z$, where $z$ is the vertical coordinate at the interface. This pressure rise must be imposed at the fluid/fluid interface; consequently, it is included in the normal stress balance, Eq. (5).

In order to fix ideas, the subscripts $/$ and $g$ are used for the liquid and gas phase respectively. Then the general jump conditions can be written as:

$$
p_{l}=p_{g}+p_{\sigma}+p_{\mu}+p_{g r}
$$

and,

$$
\tau_{l}=\tau_{g}
$$

where $p_{\sigma}, p_{\mu}, p_{\mathrm{gr}}$ are the interfacial pressure rise due to the surface curvature, the viscous normal stresses and the gravity force, respectively. In addition to the equality of the dynamically interfacial stresses described above, the kinematic conditions should also be considered. When there is no mass transfer through the interface, the kinematic conditions is satisfied by assuming the continuity of the normal velocity component, i.e.

$$
\left.\left.V_{n}\right)_{l}=V_{n}\right)_{g}
$$

The system of equations and boundary conditions (1)-(9) must be solved simultaneously to determine the flow field in the two fluids. The velocity components are then used for advection of the deformable interface by solving the appropriate equation of the level set functions.

\section{3- Level Set Formulation}

Since the original work of the level set method introduced in [18], a large amount of bibliography on the subject has been published and several types of problems have been tackled with this method; for instance one can see the cited review [19]. In the formulation of the level set, a smooth function $\phi$ is typically initialized as a signed distance function from the interface i.e. its value at any point is the distance from the nearest point on the interface and its sign is positive on one side and negative on the other. Let us set $f$ as positive in liquid and negative in gas. The location of the interface is then given by the zero level set of the function $\phi$. In absence of the interfacial mass transfer such as evaporation or condensation, the equation of the level set method can be written as: 


$$
\frac{\partial \phi}{\partial t}+\mathrm{u} \cdot \nabla \phi=0
$$

and from the level set function, the normal vector can be computed as:

$$
\mathrm{n}=-\frac{\nabla \phi}{|\nabla \phi|}
$$

By projecting the velocity $u$ onto the direction $n$ normal to the interface, and by using the definition of the normal vector, Eq. (10) becomes

$$
\frac{\partial \phi}{\partial t}+\mathrm{V}_{n}|\nabla \phi|=0
$$

A variety of methodologies have been proposed for solving the above advection equation of the level set function. One of the most recently developed numerical methods is proposed in [20]. In this method the normal velocity $V_{n}$ in Eq. (12) is replaced by some velocity field $F_{\text {ext }}$ known as the extension velocity, which at the zero level set, equals the given speed $V_{n}$. In other words,

$$
\frac{\partial \phi}{\partial t}+F_{e x t}|\nabla \phi|=0
$$

where

$$
F_{\text {ext }}=V_{n} \quad \text { on } \phi=0
$$

An important step is to maintain the level set function a distance function within the two fluids, especially near the interface region, at all times. This is achieved by the iterative reinitialization algorithm discussed in [10].

\section{NUMERICAL PROCEDURE}

Recently, we have developed a computational method for two-phase flows with a moving interface [21], in which the level set method is used to capture the moving interface. To avoid the numerical instability due to high-density ratio between the phases, each phase is treated separately, thereby; transition from one phase to another is performed through a consistent balance of kinematic and dynamic conditions on the interface separating the two phases. The accuracy of this method is examined through the computation of simple numerical examples. Furthermore, we have also confirmed the high performance of this method for convective transport simulations, in particular, the oscillatory deformations and bag break-up of a single droplet exposed in a convective gas flow, implying the high numerical accuracy and capability of this computational method to handle a wide range of incompressible two-phase flow problems.

The fundamental concepts of this numerical method are as follows: the governing transport differential equations are converted to algebraic equations before being 
solved numerically. The finite volume scheme described by [22], which involves integrating the governing equations about each control volume, yielding discrete equations, is applied. The governing equations were discretized using the secondorder central scheme to achieve the best accuracy. Pressure-velocity coupling is achieved by solving the Poisson equation for the pressure. The velocity field is assumed to reach its final value in two stages. The first stage is designed to construct a velocity field, which does not satisfy the continuity equation, based on a guessed pressure filed. The second stage is to correct the obtained velocity field for the pressure effects, whereby assuring a divergence-free velocity field.

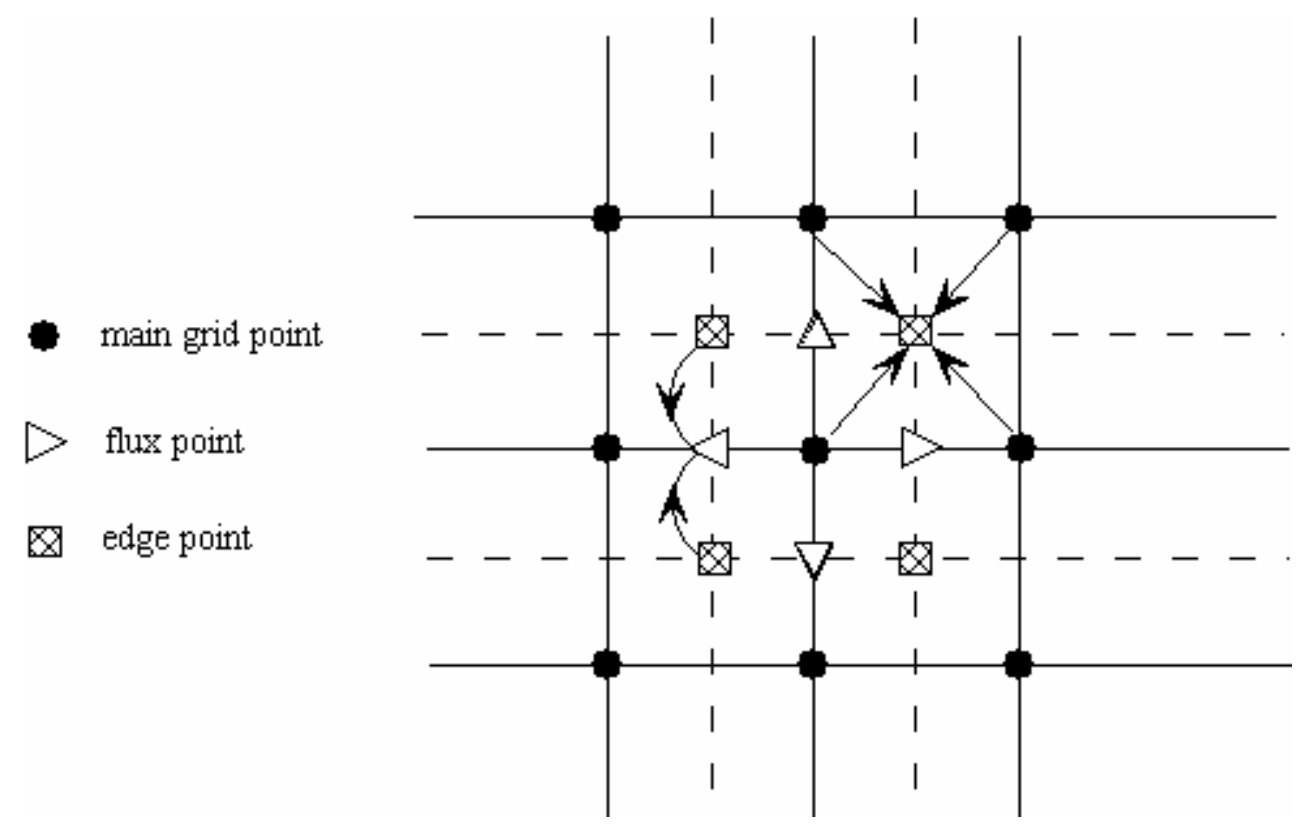

Figure (2) Calculation stencil for obtaining the intermediate flux velocity at cell faces

In case of two-fluid systems, one important requirement is the imposition of interface boundary conditions, and for this purpose a non-staggered grid is more appropriate than the staggered grid technique. The only drawback of the nonstaggered grid system is that it requires a high-order approximation to calculate the fluxes and to obtain difference equations that can prevent pressure oscillations. In the present study, the derivatives at the central point of the control volume are approximated using the second-order central difference scheme to achieve the best accuracy. The velocity components at the control volume faces are calculated using the average values of the two-edge points that are calculated through the average of the four neighbouring grid points, as seen in figure (2).

Since the interface between the two fluids is not located at the grid points, an interpolation scheme must be devised to enforce the interfacial pressure condition at the interface. The interface itself can be easily identified by locating the change in sign of $\phi$ between two contiguous points along any coordinate line. The pressure in the liquid side, derived from the jump conditions, is given by $\mathrm{Eq}$. (7) and was calculated exactly at the interface intersections with grid lines. These values are used as boundary conditions for the pressure Poisson equation. More details about the numerical algorithm and the interpolation technique can be found in [21, 23]. 
The time-stepping procedure for the level set equation is based on the secondorder Runge-Kutta method. Each time step, the level set function is reinitialized to $|\nabla \phi|=1$ in the all computational domain by applying the reinitialization algorithm described in [10] for a specified small number of iterations, see for more details [24].

\section{NUMERICAL MODEL VALIDATION}

Since the aim of this paper is to develop a numerical model for the interfacial jump conditions at an interface separating two fluids, we concentrate in different kinds of unsteady two-phase flow problems with different interfacial jump conditions, ranging from simple to complex interface topologies. To check the developed numerical modelling of the interfacial jump conditions, the numerical method described in the previous sections is applied to different unsteady cases; namely, an oscillating flow in a two-dimensional tank due to capillary force, sloshing in a twodimensional tank, a breaking dam involving different density fluids, and thermocapillary flow in two immiscible liquid layers.

\section{1- Capillary Wave Oscillation}

The wavy motion of interface separating two fluids represents one of the most important cases where capillary forces are displayed. Assuming an arbitrary, infinitesimally small, and horizontal sinusoidal displacement, as a result of random disturbances, is imposed upon the initially steady free surface. As seen in figure 3, the initial free surface has the form:

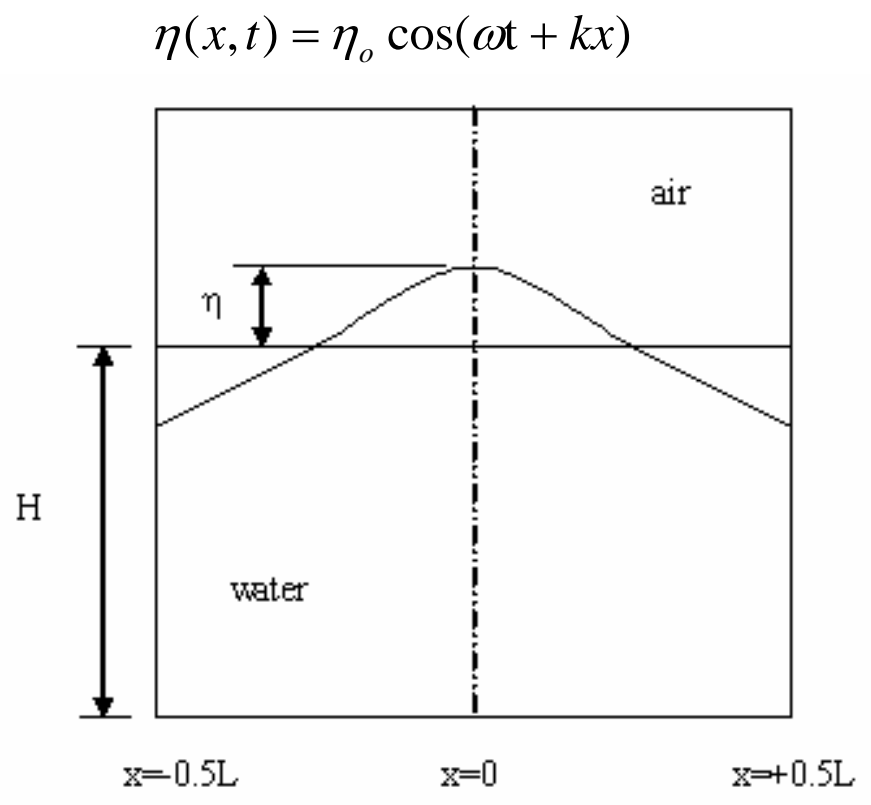

Figure (3) Schematic of flow domain configuration for capillary wave

where nrepresents the displacement of the free surface in the vertical direction from its undisturbed position, $\eta_{\mathrm{o}}$ is the initial amplitude, $\mathrm{t}$ is time, $k=2 \pi / \lambda$ is the wave number, $\lambda$ is the wavelength, and $\omega$ is the excitation frequency. The initial frequency of the oscillation is given by the following relation: 


$$
\omega^{2}=\frac{\sigma k^{3}}{2 \rho}=(13.08 \pi)^{3}
$$

Schematic of flow domain configuration is illustrated in figure 3 , where a twodimensional square tank of length $\mathrm{L}=0.7 \mathrm{~m}$ contains water of depth $\mathrm{H}=0.5 \mathrm{~m}$. The tank extends horizontally from $x=-0.5 \mathrm{~L}$ to $x=0.5 \mathrm{~L}$ and from $y=-0.5 \mathrm{~m}$ to $y=.2 \mathrm{~m}$ in the vertical direction. The water is treated as inviscid and the effect of the ambient gas on the free surface evolution is neglected. Free slip boundary conditions are applied on all boundaries. The numerical simulation is carried out over a range of wave amplitudes; $\eta_{\mathrm{o}}=0.001,0.005,0.01$. The small initial amplitudes are essentially necessary to satisfy the linear theory assumptions [25].

Figure 4 shows the capillary wave evolution at the left side and the middle of the two-dimensional tank against the non-dimensional time $t^{\star}=\omega t$ for different initial wave amplitudes. It can be seen that, no excessive damping of the free surface elevation could be observed over a large period of time. Moreover, the constant period of oscillation reveals that a good mass conservation has been obtained using the prescribed algorithm of the level set method.

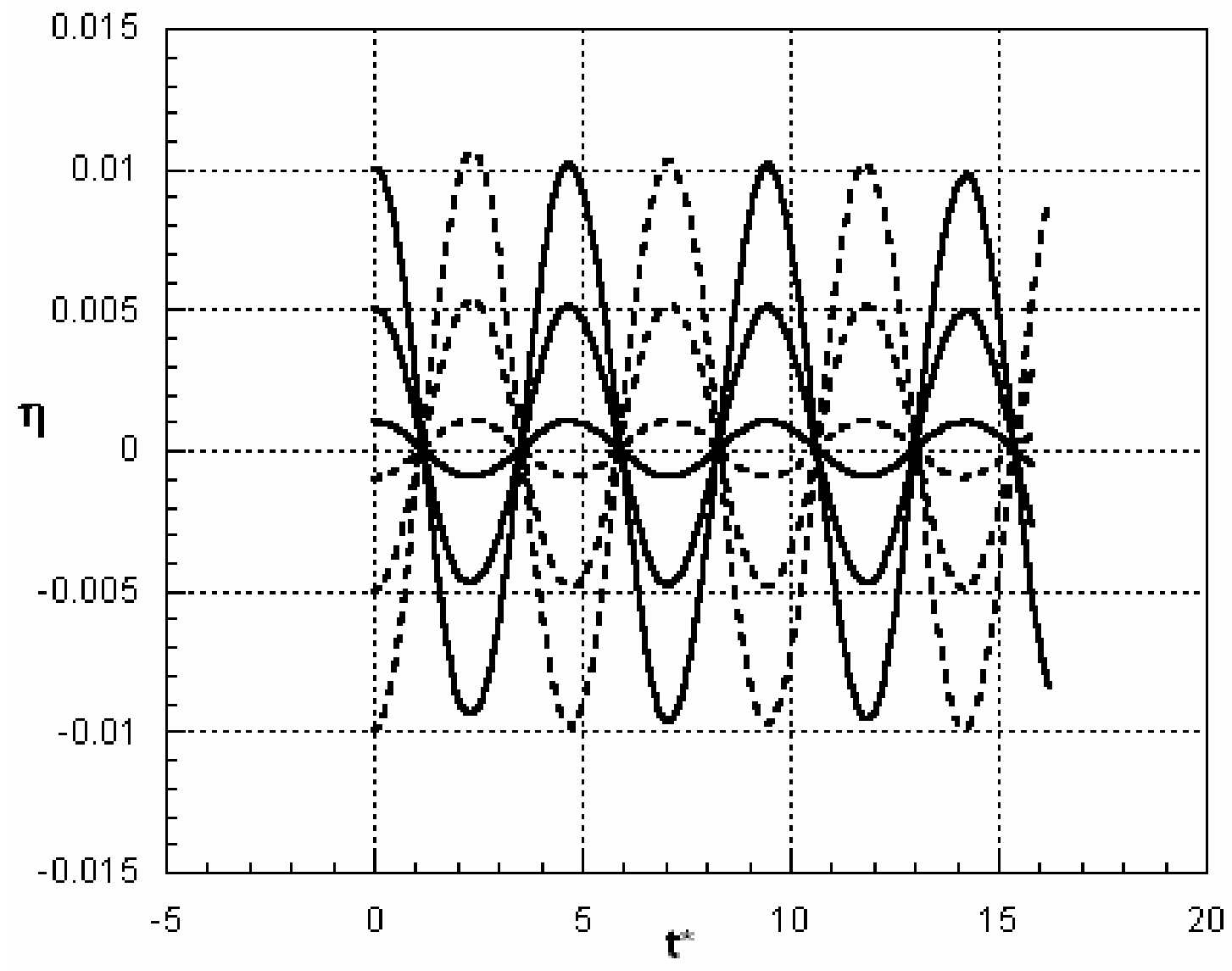

Figure (4) Time histories of the capillary wave profile at the left wall (---) and the middle (-) of the tank for different initial wave amplitudes 


\section{2- Sloshing in a Two-Dimensional Tank}

In the next examples, the present numerical method is further applied to the simulation of sloshing problem, where a free surface oscillating in a two-dimensional tank as a result of the gravity force. This example demonstrates the capabilities of both the numerical model to describe the free surface dynamics due to interfacial body forces, and the level set method to capture and to preserve mass of the free surface. For this flow, the Froude number is $\mathrm{Fr}=1$ assuming that the reference value of the velocity is $U_{\text {ref }}=(g L)^{0.5}$. The same initial and boundary conditions applied in the previous example are also applied in this example. However, the surface tension effect is neglected.

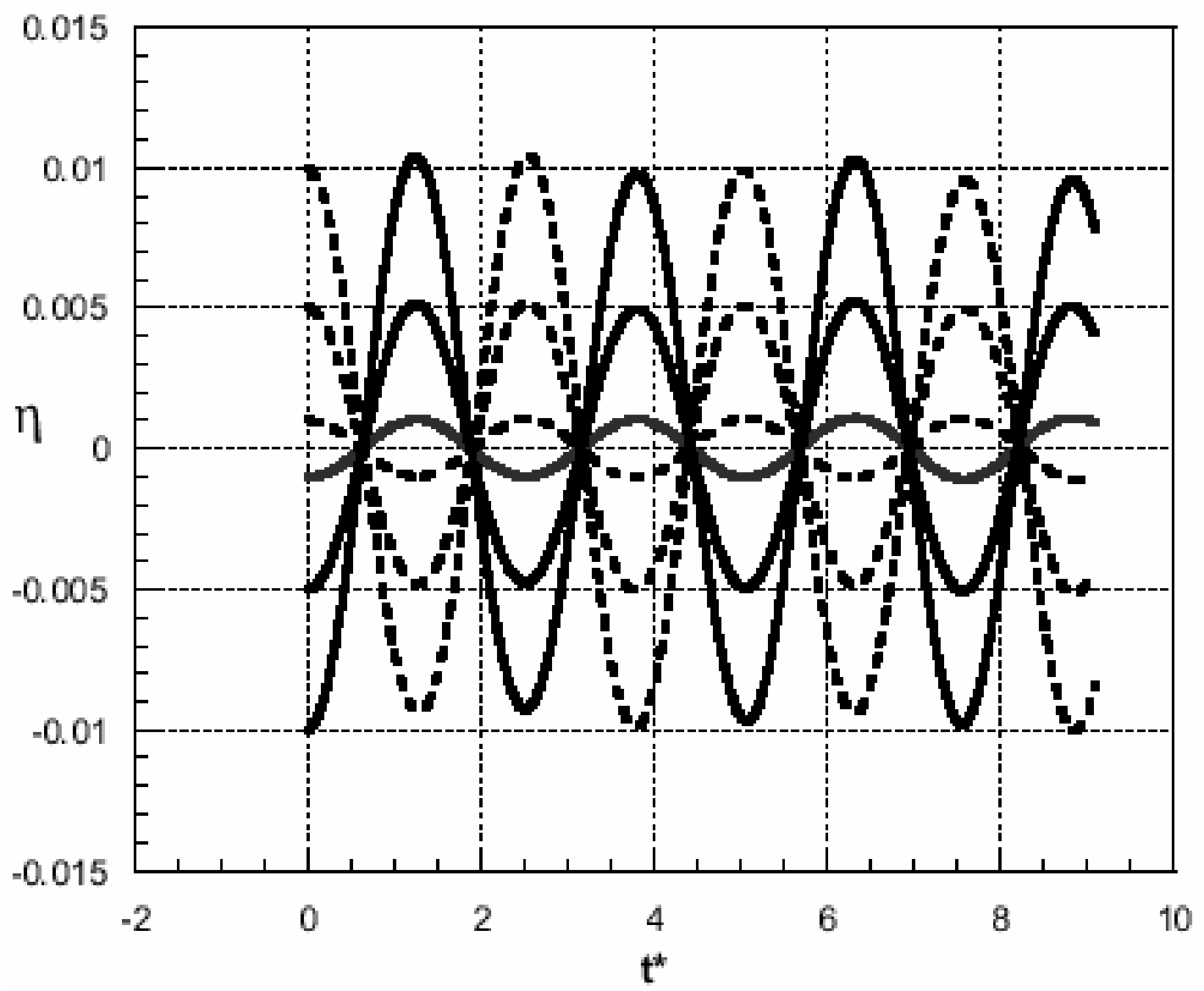

Figure (5) Time histories of the free surface amplitude for sloshing wave at the left wall (---) and the middle (-) of the tank for different initial wave amplitudes

Figure 5 shows the time evolution of the free surface elevation at the left side and at the middle of the tank for different initial disturbance amplitudes. As one can see, no excessive damping of the free surface amplitude is observed, revealing that the numerical diffusion and dissipation are diminished in the adopted scheme. In general, the present numerical method does an excellent job in predicting the capillary wave oscillation and the sloshing problem over successive oscillation periods and for different initial wave amplitudes. Thus allows an extension of the numerical model to include many physically interesting two-phase flow problems. 


\section{3- Breaking Dam Problem}

In order to further validate the present numerical algorithm, a two-dimensional broken dam problem is carried out. This test case, although of its simplicity in the initial configuration and the boundary conditions, is useful for demonstrating the versatility of the developed numerical method. In this example, a rectangular hydrostatically equilibrium water column surrounded by air is allowed to flow out along dry horizontal floor, as shown in figure 6 . The pressure jump condition at the interface is due to the gravity force acting downward, while the surface tension effect is neglected.

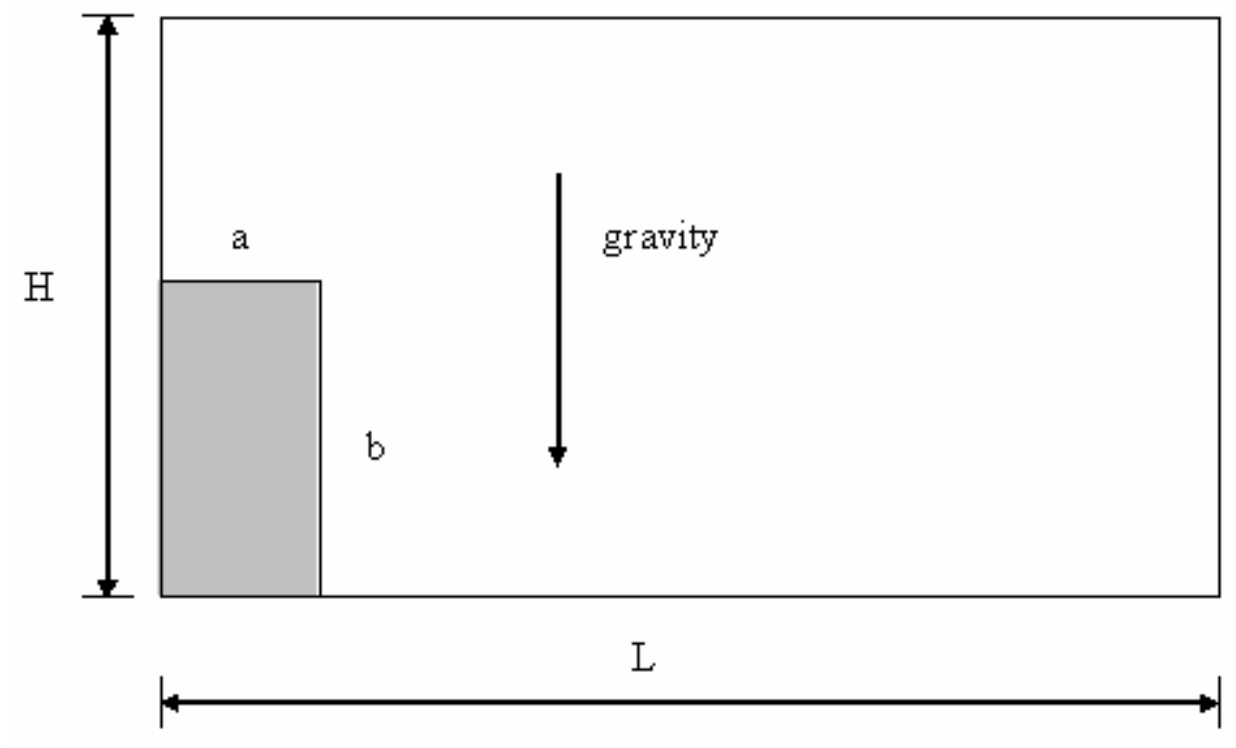

Figure (6) Configuration of broken dam problem

The rectangular computational domain with size $(\mathrm{L}=1 \mathrm{~m}, \mathrm{H}=0.5 \mathrm{~m})$ is surrounded by solid walls, at which the free-slip conditions are imposed. The water column is $0.157 \mathrm{~m}$ in width and $0.314 \mathrm{~m}$ in height, and it has the density of $1000 \mathrm{~kg} / \mathrm{m}^{3}$ and the viscous coefficient of $1.0 \times 10^{-3} \mathrm{Ns} / \mathrm{m}$, while the air has the density of $1.0 \mathrm{~kg} / \mathrm{m}^{3}$ and the viscous coefficient of $1.5 \times 10^{-5} \mathrm{Ns} / \mathrm{m}$. The computational domain has uniform grid size in $\mathrm{x}$ - and $\mathrm{y}$ - directions, respectively.

Figure 7 shows the present numerical results for the time histories of the horizontal water front location compared with both the experimental values presented in [26] and the numerical results obtained by using a novel finite-element technique [27]. The dimensionless time and displacement are given by $t^{*}=t(2 \mathrm{ga})^{0.5}$ and $x^{*}=x / a$. In the legend of this figure, the computational results are in very good agreement with the experimental ones. Further, the effects of the ratio of water-togas density on the gravity force and consequently on the horizontal location of the water front are investigated and illustrated in figure 8. The computed results show that, minor difference could be observed when the density ratio between the two fluids is more than 1:100. However, the influence of different density ratios is clearly visible. 


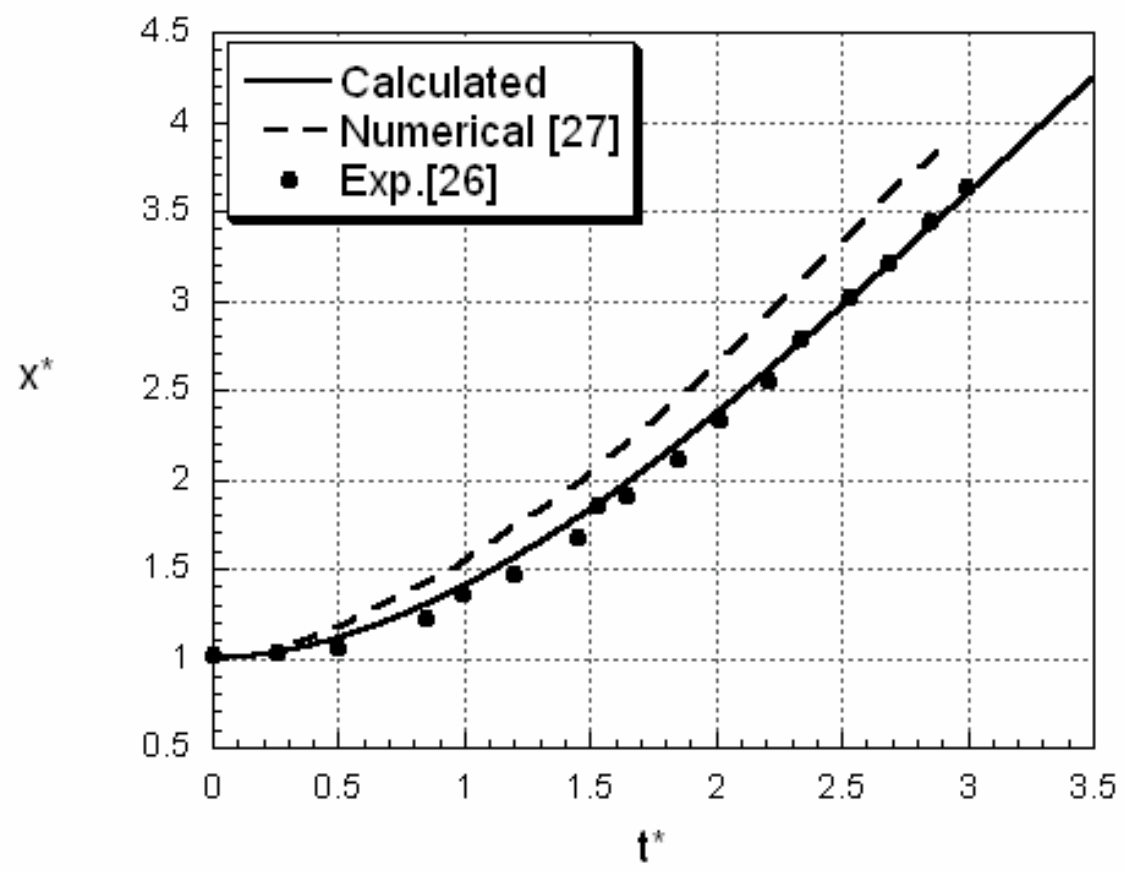

Figure (7) Comparison of calculated results for horizontal displacement of breaking dam

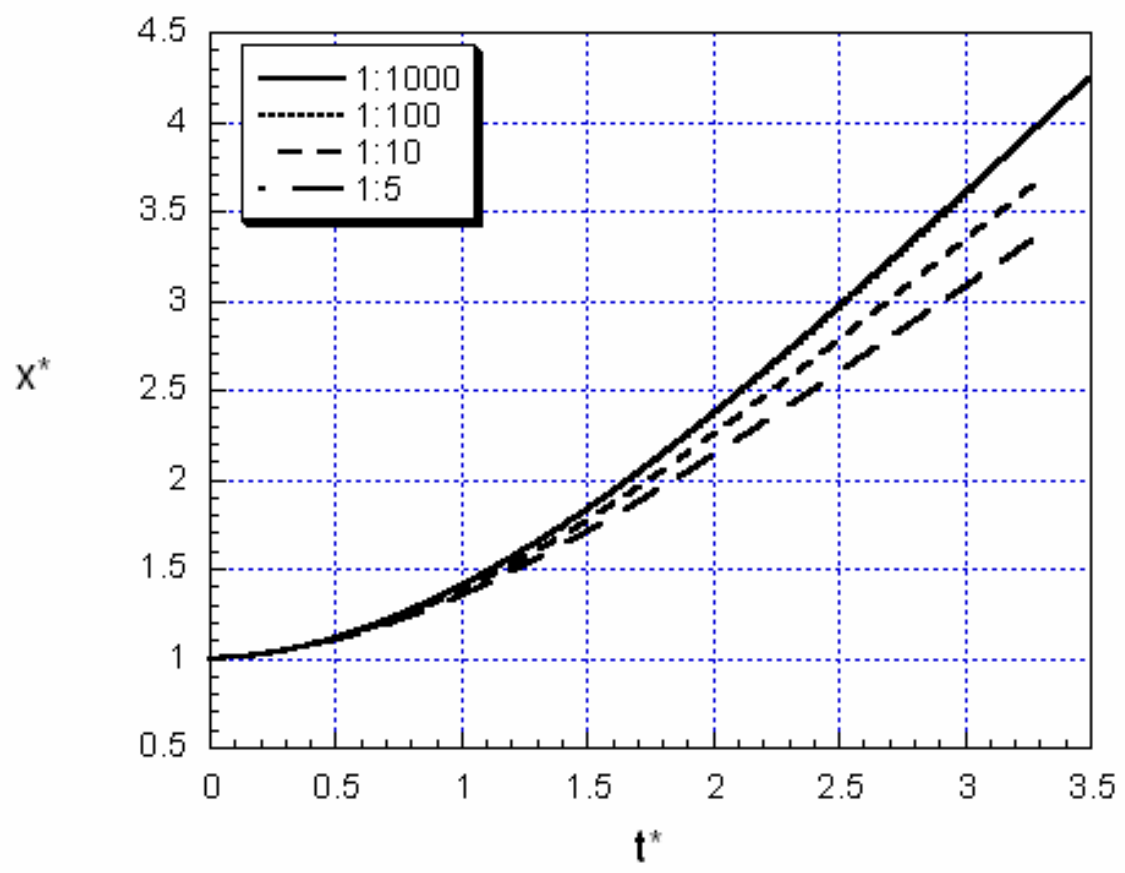

Figure (8) Horizontal water front location at different density ratios 

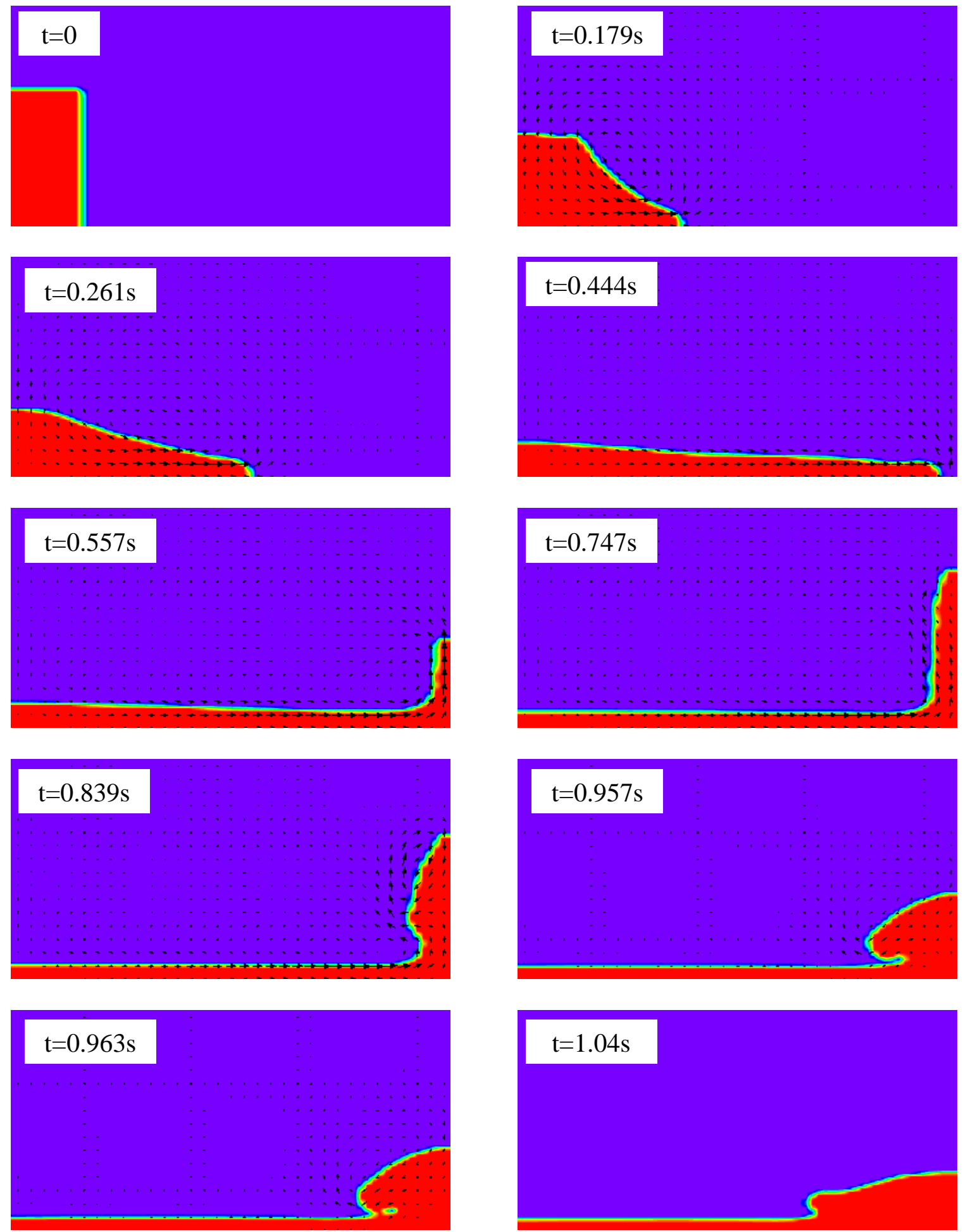

Figure (9) Fluid configuration and flow field of broken dam at different times

Figure 9 shows the computed fluid configurations and the flow field at several time levels for density ratio 1:1000. Obviously, the present method can successfully predict the topological changes of the interface and the formation of an entrapped air cavity. 


\section{CONCLUSIONS}

An accurate and robust numerical model for predicting the effects of the interfacial forces arising due to buoyancy or capillary effects in two-phase flow has been developed. The present numerical model interprets the surface and the body forces as a boundary value conditions for the pressure on the interface. The surface pressure formula works well for the interface discontinuity. The mathematical problem is formulated in primitive variables and solving using the projection method for nonstaggered grid system. The level set method has been used to present the topological changes of the interface separating the two fluids. The accuracy has been checked by applying the numerical method to the calculation of capillary and sloshing waves. The numerical diffusion and dissipation are almost vanished in the adopted scheme. The validation of the developed numerical method is confirmed through its application to the broken-dam problem and the comparison with previous experimental and numerical results. The comparison indicates that the present method give good agreement with previous experimental results and accurately satisfies the conservation of mass. The implementation of the method in three dimensions appears to be straightforward and the extension of the numerical model to include many new and physically interesting problems could be easily done.

\section{REFERENCES}

1. Eggers, J., Nonlinear Dynamics and Breakup of Free-Surface Flows, Reviews of modern Physics, Vol. 69, 3, pp. 865-929, 1997.

2. Levich, V. G. and Krylov, V.S., Surface Tension Driven Phenomena, Annu. Rev. Fluid. Mech., Vol. 1, pp. 293-316, 1969.

3. Nihei, Y. and Nadaoka, K., A Computational Method for Two-Phase Flow with a moving Boundary based on a GAL Model, Coastal Eng. Journal, Vol. 41, 3\&4, pp. 327-340, 1999.

4. Gupta, R. N., Haj-Harri, H. and Borhan, A., Thermocapillary Flow in double-layer Fluid Structures: An effective Single Layer Model, Journal of Colloid and Interface Science, Vol. 293, pp. 158-171, 2006

5. Davis, G. DE V., Natural Convection of Air in a Square Cavity: A Bench Mark Numerical Solution, Int. J. for Num. Meth. In Fluids, Vol. 3, pp. 249-264, 1983.

6. Koster, J. N. and Nguyen, KY., Steady Natural Convection in a double Layer of immiscible Liquids with Density Inversion, Int. J. Heat Mass Transfer, Vol. 39, 3, pp. 467-478, 1996.

7. Halow, F. H. and Welch, J. E., Numerical calculation of time-dependent viscous incompressible flow of fluid with free surface, Phys. Fluids, Vol. 8, pp. 21822189, 1965.

8. Hirt, C. W. and Nichols, B. D., Volume of Fluid (VOF) method for the dynamics of free boundaries, J. Comp. Physics, Vol. 39, pp. 201-225, 1981.

9. Yabe, T. and Aoki, T., A universal solver for hyperbolic equations by cubicpolynomial interpolation I. One dimensional Solver, J. Comp. Physics, Commun., Vol. 66, pp. 233-242, 1991.

10. Sussman, M. , Smereka, P. and Osher, S., A level set approach for computing solutions to incompressible two-phase flows, J. Comp. Physics, Vol. 114, pp. 146-159, 1994. 
11. Brackbill, J. U., Kothe, D. B. and Zemach, C., A continuum method for modeling surface tension, J. Comp. Physics, Vol. 100, pp. 335-354, 1992.

12. Laufaurie, B., Nardone, C., Sardovelli, R., Zaleski, S., and Zanetti, G., Modeling merging and fragmentation in multiphase flows with SURFER, J. Comp. Physics, Vol. 113, pp. 134-147, 1994.

13. Fedkiw, R. P., Aslam, T., Marriman, B., and Osher, S. J. , A non-oscillatory Eulerian approach to interface in multimaterial flows (the Ghost fluid Method), J. Comp. Physics, Vol. 154, pp. 393-427,1999.

14. Kang, M., Fedkiw, R. P. and Liu, L. D., A boundary condition capturing method for multiphase incompressible flow, J. Sci. Comp., Vol. 15, pp. 323-360, 2000.

15. Fritts, M. J., Fyfe, D. E., and Oran, E. S., Numerical simulations of droplet flows with surface tension, Proceeding of ASME workshop on Two-Phase flows, Phoenix, Arizona, 1982.

16. Tjahjadi, M., Stone, H. A., and Ottino, J. M., Satellite and subsatellite formation in capillary breakup, J. Fluid Mech., Vol.243, pp. 399-427, 1992.

17. Floryan, JM. and Rasmussen, H., Numerical methods for viscous flows with moving boundaries, App. Mech. Review, Vol. 42, pp. 323-341, 1989.

18. Osher, S. J. and Sethian, J. A., Front Propagation with curvature dependent speed: Algorithms based on Hamilton-Jacobi Formulation, J. Comp. Phys., Vol. 79, pp. 12-49, 1988.

19. Sethian, J. A. and Smereka, P., Level set methods for fluid interfaces, Annu. Rev. Fluid, Mech., Vol. 35, pp. 341-372, 2003.

20. Adalsteinsson, D. and Sethian, J. A., The fast construction of extension velocities in level set methods, J. Comp. Phys., Vol. 148, pp. 2-22, 1999.

21. Balabel, A., A computational method for immiscible two-phase flow based on the level set method.", Proceeding Of International Mechanical Engineering Conference, IMEC2004, pp. 767-786, Kuwait, 5-8 Dec. 2004.

22. Patankar, S. V., Numerical Heat Transfer and Fluid Flow, Hemisphere Publishing Corporation, 1980.

23. Balabel, A., Binninger, B., Herrmann, M. and Peters, N., Calculation of droplet deformation by surface tension effects using the level set method, Journal Combust. Sci. and Tech., Vol. 174(11,12), pp.257-278, 2002.

24. Balabel, A. and Peters, N., On the optimization of the level set method, Proceeding of the second International Mediterranean Combustion Symposium MCS02, pp. 731-738, Sharm-Elshiekh, Egypt, 2002.

25. Tadjbakhhsh, I. and Keller, J. B., Standing surface waves of finite amplitude, Journal of fluid Mechanics, Vol. 8, pp. 442-451, 1960.

26. Martin, J. C. and Moyce, W. J., An Experimental Study of the Collapse of a Liquid Column on a Rigid Horizontal Plane, Phil. Trans. Royal Soc. London A244, pp. 312-324, 1952.

27. Kölke, A., Modellierung und Diskretisierung bewegter Diskontinuitäten in Randgekoppelten Mehrfeldaufgaben, Ph.D. Thesis, TU, Braunschweig, Germany, 2005. 